\title{
Autoionizing Resonances in Time-Dependent Density Functional Theory
}

\author{
August J. Krueger ${ }^{1}$ and Neepa T. Maitra ${ }^{2}$ \\ ${ }^{1}$ Department of Physics and Astronomy, Hunter College and City University of New York, \\ 695 Park Avenue, New York, NY 10065, USA [t] \\ ${ }^{2}$ Department of Physics and Astronomy, Hunter College and The Graduate Center \\ of the City University of New York, 695 Park Avenue, New York, NY 10065, USA
}

(Dated: October 30, 2018)

\begin{abstract}
Autoionizing resonances that arise from the interaction of a bound single-excitation with the continuum can be accurately captured with the presently used approximations in time-dependent density functional theory (TDDFT), but those arising from a bound double excitation cannot. In the former case, we explain how an adiabatic kernel, which has no frequency-dependence, can yet generate the strongly frequency-dependent resonant structures in the interacting response function, not present in the Kohn-Sham response function. In the case of the bound double-excitation, we explain that a strongly frequency-dependent kernel is needed, and derive one as an a posteriori correction to the usual adiabatic approximations in TDDFT. Our approximation becomes exact for an isolated resonance in the limit of weak interaction, where one discrete state interacts with one continuum. We derive a "Fano TDDFT kernel" that reproduces the Fano lineshape within the TDDFT formalism, and also a dressed kernel, that operates on top of an adiabatic approximation. We illustrate our results on a simple model system.
\end{abstract}

\section{INTRODUCTION}

Since the birth of quantum mechanics, the study of photoionization has been important tool in characterizing the electronic structure of materials. It is desirable for theoretical methods to supplement, support, interpret, and even predict the experimental spectrum. Resonance structures, arising from the interplay of bound and continuum excitations (where this classification refers to some zeroth order model), create a fascinating panorama of peaks in the spectrum, whose profiles contain much information about the electronic states. There are three major issues to be surmounted in the theoretical treatment of resonances: first, resonances require an adequate treatment of electron correlation. We shall return to this point shortly, but note first that the considerable advances in electronic structure methods and codes for excitations over the years are predominantly set up for bound states, not continuum states. Herein lies the second issue which is adapting the many-body methods for nonsquare-integrable scattering-type states [1, 2]. Both basis set issues as well as the finite matrix-based algorithms established in quantum chemistry codes need to be revisited. When the system of interest is a molecule rather than an atom, a third ingredient compounds the problem: treating the correlated continuum states in multicenter non-spherical potentials. A variety of theoretical methods have been developed to treat these issues to a variety of extents, and we mention only a smattering of these here. For atoms, one of the more successful approaches is multi-configuration Hartree-Fock [3]; this accounts for electron correlation and core polarization accurately enough to describe atomic resonances eg.

*Electronic address: nmaitra@hunter.cuny.edu in halogens [4]. For molecules, Ref. [5] studied the relationship between interatomic distances and resonance positions using a minimal-basis static exchange method. Another approach utilizes R-matrix theory within multichannel quantum defect theory, eg. for the calcium atom in Ref. [6]. Reformulating the scattering problem as a bound-state problem in this way means that advanced electronic structure codes may be used. A complexscaled full-configuration-interaction method was used in Ref. 7] to calculate the resonances in a two-electron quantum dot. Methods using a complex absorbing potential in a configuration-interaction calculation, or with a correlated independent particle potential have been developed; an inner-valence autoionizing resonance of the neon-dimer $\mathrm{Ne}_{2}^{+}$[8], the nitrogen dimer $\mathrm{N}_{2}$ and acetylene $\mathrm{C}_{2} \mathrm{H}_{2}[9]$, for example, were computed in this way. Several other works studied autoionizing resonances in acetylene, e.g. Ref. 10] used configuration interaction within the multichannel Schwinger variational method, and Ref. [1] used $L^{2}$ Gaussian-type orbital basis sets.

Accounting for electron correlation becomes an increasingly Herculean task for wavefunction-based methods as the number of electrons in the system grows. Time-dependent density functional theory (TDDFT) [18, 19, 20] is a particularly efficient approach to the many-body problem, making it attractive for calculating photo-ionization spectra of chemically interesting systems. Recent work by Stener, Decleva, Fronzoni, and co-workers, Refs. 12, 13, 14, 15, 16, 17] (and references therein), has shown that TDDFT predicts accurate resonance parameters for a range of medium-size molecules, including acetylene, carbon monoxide, silicon tetrafluoride, and sulphur hexafluoride. Their earlier works used a one-center expansion B-spline basis, while the later ones utilized multi-centric B-spline basis functions, more suited to larger molecules.

Due to its favorable system-size scaling, TDDFT has 
become a method of choice for the calculation of bound spectra in quantum chemistry. For example, it allows calculations of spectra of systems as large as biomolecules (see eg. Refs. [23, 24, 25]), and coupled electron-ion dynamics on non-trivial chemical reactions [26]. One first computes the Kohn-Sham (KS) spectrum, which is the response of the non-interacting KS system: the single-particle excitations and oscillator strengths of the ground-state KS potential. The true spectrum is obtained by applying the linear response TDDFT exchangecorrelation (xc) kernel (see also Sec. III): operating via a matrix equation or a Dyson-type integral equation, the kernel mixes the single-excitations of the KS system, and, were the exact kernel known, this would yield the exact spectrum of the true interacting system. In practise, approximations are needed for the kernel, as well as for the ground-state KS potential out of which the KS spectrum is calculated. Of particular note for the present paper, is that almost all calculations use an adiabatic approximation to the kernel, meaning one that has no frequency-dependence.

In the TDDFT calculations of auto-ionization cited above, the role of the channel coupling is very clear: the bare KS spectra are smooth and relatively featureless, while after the TDDFT procedure is applied, resonances are generated. In one of these earliest TDDFT calculations (in the Ne atom [12]), it was noted that while the resonances arising from bound single KS excitations whose energy lie in the continuum (eg. core to Rydberg excitations) are quite accurately predicted, those arising from bound double-excitations, are totally missed. (This was also noted to occur in acetylene [14]). This was explained in Ref. [12] as arising because the linear response method involves only first-order changes in the density (or wavefunction), and therefore only single-excitations can be obtained. However, in principle, TDDFT linear response reproduces all excitations of the system, which may be linear combinations of Slater determinants with any number of excited electrons. The lack of resonances from double-excitations is not a failing of TDDFT, but rather is a failing of the approximation for the xc kernel that is used. For bound double-excitations, Ref. 27] showed that the exact kernel is necessarily stronglyfrequency-dependent in the neighbourhood of a state of double-excitation character. An approximate frequencydependent kernel was derived there to account for doubleexcitations; this was successfully tested on real molecules in Refs. 28, 29].

In the present paper, we investigate the form of the kernel that is needed in order to capture autoionizing resonances arising from a bound double-excitation. Our derivation essentially adapts Fano's 1961 analysis [30] to the case when the "unperturbed" states in his configuration mixing are the relevant KS bound-state and the continuum its energy lies in. We begin therefore, in Sec. III with a brief recapitulation of Fano's formula. In section III] we discuss the implications of Fano's formula for the density-density response functions of TDDFT, ex- plaining with an illustrative sketch, that while a strong frequency-dependence is required in the TDDFT kernel to capture resonances arising from a bound doubleexcitation, resonances arising from bound single excitations are captured by the usual adiabatic (non-frequencydependent) TDDFT kernels. Then in section IV, we derive an approximation, in the spirit of Fano, which does capture the double-excitation resonance, and illustrate it on a simple model system. Our approximation becomes exact in the limit of weak interaction, for an isolated narrow resonance in a single continuum.

\section{FANO RESONANCES}

The universality of the Fano profile has been noted by many, from lineshapes in spectra of atoms, molecules, solids and heterostructures, to interference in quantum dots and Aharanov-Bohm rings; its robustness reflected in the more than 3000 citations of his 1961 paper, Ref. [30]. The simplest type of resonance occurs when a single bound-state interacts with a single continuum. In the absence of this interaction, the continuum is assumed to be relatively "flat", i.e. featureless. Fano performed a careful diagonalization of the Hamiltonian for a continuum coupled to a single bound state $\left|\Phi_{\mathrm{b}}\right\rangle$ whose energy lies in the continuum [30]. Denoting the coupling Hamiltonian by $V_{\mathrm{cpl}}$, one defines the matrix element, $V_{E}=\left\langle\Phi_{E}\left|V_{\text {cpl }}\right| \Phi_{\mathrm{b}}\right\rangle$, with $\Phi_{E}$ being the (uncoupled) continuum state. Fano derived the following formula for the matrix element of some transition operator $\hat{T}$ (eg. a dipole operator) between an initial (bound) state $|i\rangle$ and a state $\Psi_{E}$ lying in the resonance region, resulting from the diagonalization:

$$
\frac{\left|\left\langle\Psi_{E}|\hat{T}| i\right\rangle\right|^{2}}{\left|\left\langle\Phi_{E}|\hat{T}| i\right\rangle\right|^{2}}=\frac{\left(\omega-\omega_{r}+\Gamma q / 2\right)^{2}}{\left(\omega-\omega_{r}\right)^{2}+(\Gamma / 2)^{2}}
$$

Atomic units are used throughout this paper. Here $\omega=$ $E-E_{i}$ is the frequency, and

$$
\omega_{r}=E_{r}-E_{i}=E_{\mathrm{b}}-E_{i}+P \int \frac{\left|V_{E^{\prime}}\right|^{2}}{E-E^{\prime}} d E^{\prime}
$$

is the "position" of the resonance, shifting the unperturbed bound-state frequency $E_{\mathrm{b}}-E_{i}$ by the principalvalue integral. The parameter

$$
\Gamma=2 \pi\left|V_{E}\right|^{2}
$$

defines the width of the resonance, while the parameter $q$ characterizes its asymmetry:

$$
q=\frac{\left\langle\Phi_{\mathrm{b}}|\hat{T}| i\right\rangle+P \int V_{E^{\prime}}\left\langle\Phi_{E^{\prime}}|\hat{T}| i\right\rangle /\left(E-E^{\prime}\right) d E^{\prime}}{\pi V_{E}\left\langle\Phi_{E}|\hat{T}| i\right\rangle}
$$

For example, $q=0$ represents a negative purely symmetric Lorentzian, $q \rightarrow \infty$ represents a positive purely symmetric Lorentzian, and $q= \pm 1$ represents a purely 
antisymmetric lineshape. The asymmetry can be interpreted as a consequence of interference between the autoionizing state and the continuum states [30, 31, 32]. In Refs. [30, 32], it is argued that typically $q$ is negative.

Although Eqs. (3) and (41) appear energy-dependent, $q, \Gamma$, and $\omega_{r}$ are regarded as constant through the resonance region. For a narrow enough resonance, this is a reasonable assumption; essentially the idea is that $\Gamma$ is the smallest energy scale in the system. Fano also derived a "sum-rule" for the integrated transition probability:

$$
\begin{aligned}
\left|\left\langle\Phi_{\mathrm{b}}|T| i\right\rangle\right|^{2} & =\int d E\left(\left|\left\langle\Psi_{E}|T| i\right\rangle\right|^{2}-\left|\left\langle\Phi_{E}|T| i\right\rangle\right|^{2}\right) \\
& =\left|\left\langle\Phi_{E}|T| i\right\rangle\right|^{2} \frac{\pi}{2}\left(q^{2}-1\right) \Gamma
\end{aligned}
$$

which expresses the unitary nature of the diagonalization procedure. Eq. 5 is essentially a consequence of the following closure relation:

$$
\int\left|\Psi_{E}\right\rangle\left\langle\Psi_{E}\left|d E=\int\right| \Phi_{E}\right\rangle\left\langle\Phi_{E}|d E+| \Phi_{\mathrm{b}}\right\rangle\left\langle\Phi_{\mathrm{b}}\right|
$$

Fano's Eq. (1) tells us that the transition to the continuum when a discrete state couples to the continuum, is equal to that without the coupling, multiplied by a (generally asymmetric) Lorentzian line-shape factor.

Photoabsorption cross-sections measure the dipole transition probability, so take $\hat{T}$ to be the dipole operator. Fits are routinely made for the Fano parameters $q, \Gamma$ and $\omega_{r}$ for a given cross-section obtained from experiment or theory, i.e. Eqs. (2) - 4) are not typically used to calculate these quantities, rather, they are extracted from experimental or theoretical data. Although we will only use the simplest type of resonance in the present paper, we do note that Fano's analysis has been generalized in several directions [30, 32, 33], e.g. when more continua are present. The more complicated situations involve more fitting parameters, and in practise fits are made for these more general formulae, rather than the simplest situation discussed above.

\section{AUTOIONIZING RESONANCES WITHIN ADIABATIC TDDFT}

\section{A. Photoabsorption/ionization in TDDFT}

The photoabsorption or photoionization cross-section measures the linear density response $\delta n(\mathbf{r}, \omega)$ of a system subject to an external electric field of frequency $\omega$. Letting $\alpha, \beta$ denote the three spatial directions, the crosssection tensor is

$$
\sigma_{\alpha \beta}(\omega)=\frac{4 \pi \omega}{c} \int d^{3} r d^{3} r^{\prime} r_{\alpha} r_{\beta}^{\prime} \operatorname{Im} \chi\left(\mathbf{r}, \mathbf{r}^{\prime}, \omega\right)
$$

where $\chi\left(\mathbf{r}, \mathbf{r}^{\prime}, t-t^{\prime}\right)=\delta n(\mathbf{r}, t) / \delta v_{\text {ext }}\left(\mathbf{r}^{\prime}, t\right)$ is the densitydensity response function of a system to an external potential $v_{\text {ext }}(\mathbf{r}, t)$ (see, eg. Refs. [34, 35]). A sum-overstates expression may be obtained using the standard linear response theory expansion for the density-density response function:

$\chi\left(\mathbf{r}, \mathbf{r}^{\prime}, \omega\right)=\sum_{E} \frac{\langle 0|\hat{n}(\mathbf{r})| E\rangle\left\langle E\left|\hat{n}\left(\mathbf{r}^{\prime}\right)\right| 0\right\rangle}{\omega-\left(E-E_{0}\right)+i 0^{+}}-\frac{\left\langle 0\left|\hat{n}\left(\mathbf{r}^{\prime}\right)\right| E\right\rangle\langle E|\hat{n}(\mathbf{r})| 0\rangle}{\omega+\left(E-E_{0}\right)+i 0^{+}}$

where $|E\rangle$ label the excited states, and $|0\rangle$ is the groundstate with energy $E_{0} . \hat{n}(\mathbf{r})=\sum_{i}^{N} \delta\left(\mathbf{r}-\mathbf{r}_{i}\right)$ is the density operator. Assuming real eigenstates, the imaginary part is extracted as

$$
\begin{aligned}
\Im \chi\left(\mathbf{r}, \mathbf{r}^{\prime}, \omega\right)= & -\pi \sum_{E}\langle 0|\hat{n}(\mathbf{r})| E\rangle\left\langle E\left|\hat{n}\left(\mathbf{r}^{\prime}\right)\right| 0\right\rangle \times \\
& \left(\delta\left(\omega-\omega_{E}\right)-\delta\left(\omega+\omega_{E}\right)\right)
\end{aligned}
$$

where $\omega_{E}=E-E_{0}$. Inserting this into Eq. 17 we have

$$
\begin{aligned}
\sigma_{\alpha \beta}(\omega)= & -\frac{4 \pi^{2} \omega}{c} \sum_{E}\left\langle 0\left|\hat{\mathbf{r}}_{\alpha}\right| E\right\rangle\left\langle E\left|\hat{\mathbf{r}}_{\beta}^{\prime}\right| 0\right\rangle \times \\
& \left(\delta\left(\omega-\omega_{E}\right)-\delta\left(\omega+\omega_{E}\right)\right) \\
= & -\frac{4 \pi^{2} \omega}{c} \sum_{E} d_{\alpha}(\omega) d_{\beta}(\omega)\left(\delta\left(\omega-\omega_{E}\right)-\delta(\omega+\omega(10))\right.
\end{aligned}
$$

where $d_{\alpha}(\omega)$ is the transition dipole moment from the ground-state to the excited state of energy $E=\omega+E_{0}$.

Therefore, to compute photoabsorption or photoionization, one needs an efficient way to calculate either the density response $\delta n(\mathbf{r}, \omega)$, or the density-density response function, $\chi\left(\mathbf{r}, \mathbf{r}^{\prime}, \omega\right)$ (Eq. 7) or the excited states of the system (Eq. 10). Given that the electrons in the system are interacting with all the others via Coulomb repulsion, this becomes a daunting task for correlated wavefunction methods for all but the smallest molecules: the numerical effort in solving the problem scales exponentially with the number of electrons in the system.

In TDDFT, instead of dealing with the correlated many-body wavefunction, one solves for the much simpler single-particle orbitals $\phi_{i}(\mathbf{r}, t)$ that evolve in the onebody KS Hamiltonian. Nevertheless, from the $\phi_{i}(\mathbf{r}, t)$ in principle, not just the exact density but all properties of the true interacting correlated system may be extracted [18, 20]. In practise approximations are needed for the xc terms.

Linear response TDDFT [21, 22] is founded on the relation between the interacting and KS density-response functions, both of which are functionals of the groundstate density of the system of interest, $n_{0}(\mathbf{r})$. First, we note that for non-interacting systems such as KS, the numerator in Eq. 8 simplifies to products of occupied (indexed by $i$ ) and unoccupied (indexed by $a$ ) orbitals:

$\chi_{\mathrm{S}}\left(\mathbf{r}, \mathbf{r}^{\prime}, \omega\right)=\sum_{i, a} \frac{\Phi_{i a}(\mathbf{r}) \Phi_{i a}^{*}\left(\mathbf{r}^{\prime}\right)}{\omega-\left(\epsilon_{a}-\epsilon_{i}\right)+i 0^{+}}-\frac{\Phi_{i a}^{*}(\mathbf{r}) \Phi_{i a}\left(\mathbf{r}^{\prime}\right)}{\omega+\left(\epsilon_{a}-\epsilon_{i}\right)+i 0^{+}}$

where $\Phi_{i a}(\mathbf{r})=\phi_{i}^{*}(\mathbf{r}) \phi_{a}(\mathbf{r})$. The orbital energydifferences in the denominator $\epsilon_{a}-\epsilon_{i}$, are the KS singleexcitation frequencies, and the orbitals live in the KS 
potential $v_{\mathrm{S}}(\mathbf{r})=v_{\text {ext }}(\mathbf{r})+v_{\mathrm{H}}(\mathbf{r})+v_{\mathrm{XC}}(\mathbf{r})$. Here $v_{\mathrm{H}}(\mathbf{r})=$ $\int d^{3} r^{\prime} n\left(\mathbf{r}^{\prime}\right) /\left|\mathbf{r}-\mathbf{r}^{\prime}\right|$ is the Hartree potential, and $v_{\mathrm{XC}}(\mathbf{r})$ is the (ground-state) xc potential. The frequencies of the true system lie at the poles of the true interacting response function, $\chi\left(\mathbf{r}, \mathbf{r}^{\prime}, \omega\right)$ :

$$
\begin{gathered}
\chi\left[n_{0}\right]\left(\mathbf{r}, \mathbf{r}^{\prime}, \omega\right)=\chi_{\mathrm{S}}\left[n_{0}\right]\left(\mathbf{r}, \mathbf{r}^{\prime}, \omega\right)+ \\
\left.\int d r_{1} d r_{2} \chi_{\mathrm{S}}\left[n_{0}\right]\left(\mathbf{r}, \mathbf{r}_{1}, \omega\right) f_{\mathrm{HXC}}\left[n_{0}\right]\left(\mathbf{r}_{1}, \mathbf{r}_{2}, \omega\right) \chi\left[n_{0}\right]\left(\mathbf{r}_{2}, \mathbf{r}^{\prime}, \omega\right) 12\right)
\end{gathered}
$$

where $f_{\mathrm{HXC}}$ denotes the "Hartree-exchange-correlation kernel",

$$
\begin{array}{r}
f_{\mathrm{HXC}}\left[n_{0}\right]\left(\mathbf{r}, \mathbf{r}^{\prime}, \omega\right) \equiv \frac{1}{\left|\mathbf{r}-\mathbf{r}^{\prime}\right|}+f_{\mathrm{XC}}\left[n_{0}\right]\left(\mathbf{r}, \mathbf{r}^{\prime} \omega\right) \\
=\chi_{\mathrm{S}}^{-1}\left[n_{0}\right]\left(\mathbf{r}, \mathbf{r}^{\prime}, \omega\right)-\chi^{-1}\left[n_{0}\right]\left(\mathbf{r}, \mathbf{r}^{\prime}, \omega\right)
\end{array}
$$

with $f_{\mathrm{XC}}\left[n_{0}\right]\left(\mathbf{r}, \mathbf{r}^{\prime}, t-t^{\prime}\right)=\delta v_{\mathrm{XC}}\left[n_{0}\right](\mathbf{r}, t) / \delta n\left(\mathbf{r}^{\prime}, t\right)$.

Although the exact xc kernel, $f_{\mathrm{XC}}(\omega)$ is frequencydependent, reflecting a dependence on the history of the density in the time-domain [36], the majority of calculations today utilize an "adiabatic" approximation, meaning one where $v_{\mathrm{xC}}[n](r, t)$ depends only on the instantaneous density $f_{\mathrm{xC}}^{A}\left[n_{0}\right]\left(\mathbf{r}, \mathbf{r}^{\prime}, t-t^{\prime}\right) \propto \delta\left(t-t^{\prime}\right)$. When Fourier-transformed, this gives no structure in frequencyspace. Instead, the adiabatic approximation is based on a ground-state energy functional

$$
f_{\mathrm{XC}}^{A}\left[n_{0}\right]\left(\mathbf{r}, \mathbf{r}^{\prime}\right)=\left.\frac{\delta^{2} E_{\mathrm{XC}}[n]}{\delta n\left(\mathbf{r}^{\prime}\right) \delta n(\mathbf{r})}\right|_{n=n_{0}}
$$

Despite incorrectly lacking frequency-dependence, the adiabatic approximation yields remarkably accurate results for most excitations. Eq. 12 can be transformed to a matrix equation indexed by KS single-excitations [22], the workhorse of bound-state TDDFT. When the true interacting state is composed of mixtures of $\mathrm{KS}$ single excitations, adiabatic TDDFT is expected to work reasonably well - provided the spatial functional dependence of $v_{\mathrm{xC}}(\mathbf{r})$ is adequately nonlocal for the problem at hand. For states of multiple-excitation character however, one must go beyond the adiabatic approximation as was shown for bound states of finite systems [27, 28, 29, 37, 38], as mentioned in the introduction. We shall show shortly, that when such a state lies in the continuum, a frequency-independent kernel misses the resonance arising from it.

\section{B. Autoionizing Resonances in TDDFT}

In photo-ionization, the energy of interest lies in the continuum; the sum over the delta-function peaks in Eqs. (9) and (10) becomes an integral, and, (for positive $\omega$, greater than the ionization threshold),

$\Im \chi\left(\mathbf{r}, \mathbf{r}^{\prime}, \omega\right)=-\pi\left\langle 0|\hat{n}(\mathbf{r})| E=\omega+E_{0}\right\rangle\left\langle E=\omega+E_{0}\left|\hat{n}\left(\mathbf{r}^{\prime}\right)\right| 0\right\rangle$ where the continuum states are chosen real and energynormalized. The KS cross-section for continuum transitions are largely structureless, especially when only one continuum is relevant, the $\mathrm{KS}$ transition dipole is:

$$
\mathbf{d}_{\mathrm{S}}(\omega)=\left\langle\phi_{\epsilon=\epsilon_{i}+\omega}|\mathbf{r}| \phi_{i}\right\rangle
$$

where $\phi_{i}$ is the KS occupied orbital, of energy $\epsilon_{i}$, out of which excitation occurs to the continuum orbital $\phi_{\epsilon}$, of energy $\epsilon=\epsilon_{i}+\omega$. This typically gently decays as a function of frequency, reflecting the decay of the overlap between an occupied orbital and a continuum one as the energy of the continuum state rises.

When one applies TDDFT to obtain the spectrum of the interacting system, the kernel $f_{\mathrm{HXC}}$ mixes the KS single excitations and the spectrum distorts to varying degrees: less at the higher frequencies, and most significantly near resonances. The TDDFT kernel smears the oscillator strength from bound transitions whose energy lies in the continuum over a narrow range in the continuum, implicitly performing the job of the Fano diagonalization of Section [I] Effectively, a rather featureless KS continuum spectrum is turned into a dramatically frequency-dependent interacting spectrum, via the operation of the xc kernel. Refs. [12, 13, 14, 15, 16, 17] have demonstrated this explicitly on a wide range of interesting atoms and molecules. These works show that adiabatic kernels reproduce resonance features of interacting systems rather well through this action, when the resonances arise from a bound single excitation with energy lying in the continuum (e.g. of core-Rydberg nature).

The appearance of the resonance can therefore be understood as a mixing of the single excitations appearing in Eq. (11) via Eq. (12). Yet, how a frequencyindependent kernel can transform the largely frequencyindependent KS continuum spectrum into a spectrum that does have such dramatic frequency-dependence, may strike one as incongruous. To understand this better, we first find an expression relating the interacting and $\mathrm{KS}$ response functions near an autoionizing resonance, following in Fano's footsteps.

We keep to the simple case of one discrete state lying in one continuum. Further, we consider, initially, only the resonant coupling; that is, we treat the KS states as Fano's "pre-diagonalized" states, without accounting for mixing amongst them. Although this could only be the case when there is no electron-interaction, the justification for this simplification is that in the vicinity of the resonance, this resonant coupling is certainly the dominant effect: the coupling amongst the KS states is an order of $\Gamma$ less. (Later, in Sec IV] we relax this assumption). For the matrix elements on the right-hand-side of we use Eq. (1), but taking $\hat{T}$ as the density operator $\hat{n}(\mathbf{r})$ :

$$
\langle 0|\hat{n}(\mathbf{r})| E\rangle=\sqrt{\frac{\left(\omega-\omega_{r}+\Gamma q(\mathbf{r}) / 2\right)^{2}}{\left(\omega-\omega_{r}\right)^{2}+(\Gamma / 2)^{2}}}\langle 0|\hat{n}(\mathbf{r})| E\rangle_{\mathrm{S}}
$$

where the ket on the right is the KS continuum excited state at energy $E=\omega+E_{0}$. The state $|0\rangle$ on both sides 
of the equation is the initial state out of which transitions occur, which we will take to be the ground-state. Technically, this should be the interacting ground-state because Fano's analysis assumed everything except for the resonant coupling was in the zero-order states. However, if we approximate the bra $\langle 0|$ on the right of Eq. (17) to be instead the KS ground-state, this formula then directly relates the interacting matrix element (related to the oscillator strength) to the KS matrix element. This approximation holds if the KS ground-continuum transition in the absence of the resonance is a good approximation to the true interacting transition, and again, holds under the justification that the resonant coupling is the dominant effect in the resonance region.

The quantities $\Gamma, q$ and $\omega_{r}$ are given by Eq. 3, 4, and 2, where the "coupling" $V_{\text {cpl }}$ is the difference in the Hamiltonian of the true and KS systems:

$$
V_{\mathrm{cpl}}=V_{\mathrm{ee}}-v_{\mathrm{H}}-v_{\mathrm{XC}}
$$

where $V_{\mathrm{ee}}=\frac{1}{2} \sum_{i j} 1 /\left|\mathbf{r}_{i}-\mathbf{r}_{j}\right|$ is the electron-electron interaction. Inserting Eq. 17 into Eq. 15] for $\omega$ near a resonance,

$$
\begin{aligned}
\Im \chi\left(\mathbf{r}, \mathbf{r}^{\prime}, \omega\right) & =\frac{\left(\omega-\omega_{r}+\Gamma q(\mathbf{r}) / 2\right)\left(\omega-\omega_{r}+\Gamma q\left(\mathbf{r}^{\prime}\right) / 2\right)}{\left(\omega-\omega_{r}\right)^{2}+(\Gamma / 2)^{2}} \\
& \times \Im \chi_{S}^{c}\left(\mathbf{r}, \mathbf{r}^{\prime}, \omega\right)
\end{aligned}
$$

where $\chi_{\mathrm{S}}^{\mathrm{c}}\left(\mathbf{r}, \mathbf{r}^{\prime}, \omega\right)$ denotes the continuum contribution to the KS response function at frequency $\omega$. Following directly from Fano's analysis, Eq. 19 is a new relation between the true and KS response functions in the neighborhood of any isolated narrow autoionizing resonance, that arises from the mixing of a single discrete state with a single continuum, under the assumption of weak interaction. The real part of the response function can be obtained via the principle-value integral:

$$
\Re \chi\left(\mathbf{r}, \mathbf{r}^{\prime}, \omega\right)=\frac{2}{\pi} P \int_{0}^{\infty} \frac{\omega^{\prime} \Im \chi\left(\mathbf{r}, \mathbf{r}^{\prime}, \omega\right)}{\omega^{\prime 2}-\omega^{2}} d \omega^{\prime}
$$

due to known analyticity properties of $\chi$ (eg. Ref. [35]).

We now address the curiousity raised earlier regarding how application of a frequency-independent adiabatic kernel to the flat KS spectrum can generate frequencydependent resonant structure in $\chi$. A simple sketch is instructive to show this. Consider a resonance due to a bound single-excitation, at frequency $\omega_{b}>I$, the ionization potential. Within our weak-interaction assumption the KS single excitation couples only to the continuum states, so, near $\omega_{\mathrm{b}}, \chi_{\mathrm{s}}$ has the form

$$
\chi_{\mathrm{S}}(\omega)=\chi_{\mathrm{S}}^{\mathrm{c}}(\omega)+\chi_{\mathrm{S}}^{\mathrm{b}}(\omega)
$$

where $\chi_{\mathrm{S}}^{\mathrm{c}}(\omega)$ is complex, smooth and gently-decaying and $\chi_{\mathrm{S}}^{\mathrm{b}}(\omega)$ is the contribution of the bound state to the sum over states Eq. (11). For the present illustrative purposes, we neglect the spatial-dependence. We take $\chi_{\mathrm{S}}^{\mathrm{c}}(\omega)=\left(\frac{1}{\sqrt{\omega+1}}+\frac{i}{\sqrt{\omega-1}}\right)$ in the plots, although our conclusions in no way depend on this form, and $\chi_{\mathrm{S}}^{\mathrm{b}}(\omega)=\frac{b}{\omega-\omega_{\mathrm{b}}+i 0^{+}}$. Here $b$ represents the orbital products appearing in the residue of Eq. (11). The imaginary part of this $\chi_{\mathrm{S}}(\omega)$ is plotted as the solid line on the top left panel of Figure 1. In particular, note that $\Im \chi_{\mathrm{s}}$, which is directly related to the measured cross-section (Eq. (7), has the structureless continuum contribution plus a deltapeak (indicated by the arrow) at $\omega_{\mathrm{b}}$ (taken to be 3 , while $b$ is taken to be -0.01 in these plots and $I=0.5 \mathrm{au}$ ); other values yield similar plots). (Note that the delta-peak is not evident in the smooth $\mathrm{KS}$ cross-sections plotted in the graphs of Refs. [12, 13, 14, 15, 16, 17], because there only the KS continuum transitions in $\chi_{\mathrm{S}}^{\mathrm{c}}$ (Eq. (16)) are included). Now consider inverting Eq. 21. Interestingly, this immediately displays resonance-structures in both the real and imaginary part of $\chi_{\mathrm{S}}^{-1}(\omega)$, as shown by the solid lines on the right-hand panel of Figure 1. This may be simply seen mathematically: inverting Eq. 21 reveals a Lorentizan denominator in both the real and imaginary parts. Applying the TDDFT kernel, to obtain $\chi^{-1}=\chi_{\mathrm{S}}^{-1}-f_{\mathrm{HXC}}$ (Eq. (13)), we see that an adiabatic approximation $f_{\mathrm{HXC}}^{A}$ (Eq. (14)) just uniformly shifts the real part of $\chi_{\mathrm{S}}^{-1}$, while not adding any additional frequencydependence. Indeed the resonance structure of $\chi_{\mathrm{S}}^{-1}$ and the adiabatically shifted one $\left(\chi^{A}\right)^{-1}$ resemble that of the Fano profile of Eq. (19): for a constant $q=-3$, the inverse of $\chi$ computed from Eqs. (19) 20) is plotted in the inset on the right. No frequency-dependence is required in the kernel itself to obtain resonances in $\chi_{\mathrm{S}}$. We now invert $\left(\chi^{A}\right)^{-1}$ to obtain $\left(\chi^{A}\right)$, plotted back as the dashed curves in the left-hand panels of Figure 1 These resemble the Fano profile of Eq. (19) for $q=-3$ plotted in the insets on the left panels. The adiabatic shift in the inverse response functions, had the effect of Lorentzian smoothing the delta-function peak in $\Im \chi_{\mathrm{s}}$, producing a resonance in $\Im \chi^{A}$. Therefore, making a adiabatic shift in the real part of the inverse response fn, $\Re \chi_{\mathrm{S}}^{-1}$, turns, upon inversion, a delta-peak of $\Im \chi_{\mathrm{S}}$ into a Lorentzian resonance peak of $\Im \chi^{A}$. In this way, inversion of $\chi_{\mathrm{S}}^{-1}$, shifted frequency-independently, generates frequency-dependent structure in $\chi$.

For a resonance arising from a double-excitation however, the analogous figures clearly show that frequencydependence in the kernel is absolutely required. The KS response function of Eq. 21] now consists solely of the gently decaying first term, $\chi_{\mathrm{S}}^{\mathrm{c}}$, (left-hand panel of Figure 2) i.e. there is no bound-state contribution to $\chi_{\mathrm{S}}$ because a double-excitation has zero oscillator strength [27]. The true response function however displays a resonance with an antisymmetric imaginary part $(q= \pm 1)$ in order to preserve the oscillator strength sum-rule (see Sec. [II). On the right panel of Figure 2 we invert this smooth $\chi_{\mathrm{s}}$ to obtain the featureless $\chi_{\mathrm{S}}^{-1}$ shown (solid lines). The dotted curves represent the true $\chi^{-1}$, computed using Eq. (19) for $q=1$. A frequency-independent kernel again only shifts the real part of $\chi_{\mathrm{S}}^{-1}$ uniformly as shown, but in this case it cannot generate the resonance-structure of the true $\chi^{-1}$, shown as dotted lines in the figure. For that, 

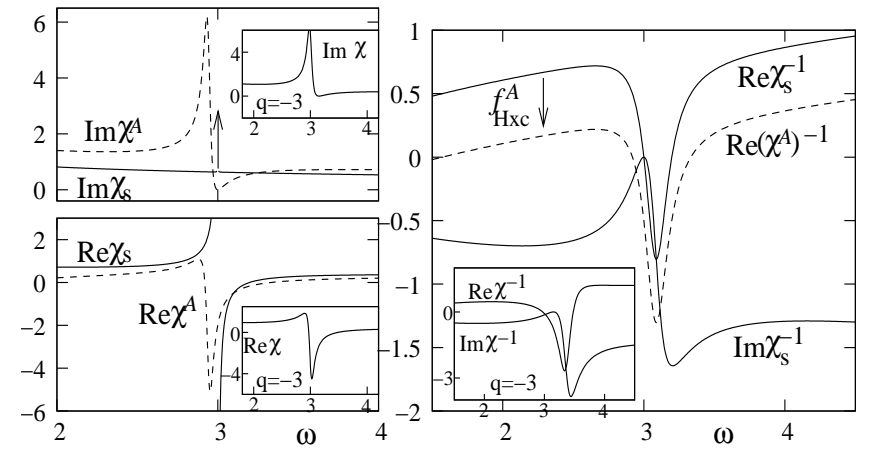

FIG. 1: The imaginary and real parts of $\chi_{\mathrm{S}}(\omega)$ (left-hand panels, solid lines). The delta-function in the imaginary part of $\chi_{\mathrm{S}}$ corresponding to the bound-state, is shown as an arrow. On the right, we show $\chi_{\mathrm{S}}^{-1}$ (solid) and the shift that an adiabatic kernel $f_{\mathrm{HXC}}^{A}$ produces, yielding $\left(\chi^{A}\right)^{-1}$ (dashed). When inverted to yield the adiabatic response kernel $\chi^{A}(\omega)$, the resonance emerges, as indicated by the dashed lines on the left panels. The adiabatic curves should be compared with the insets, which come from Eq. 19 with $q=-3$, see text

a frequency-dependent kernel of the form derived in the next section is required. The left-hand panel shows the real and imaginary parts of $\chi_{\mathrm{s}}$ and $\chi^{A}$, lacking resonance.

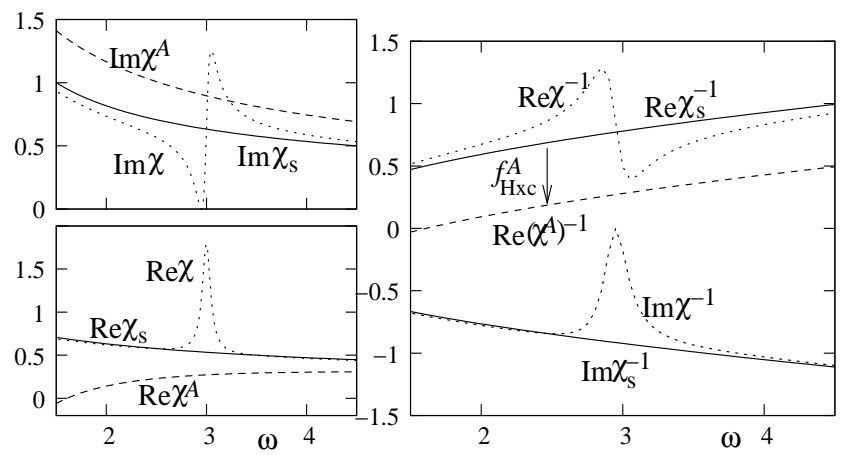

FIG. 2: The imaginary and real parts of $\chi_{\mathrm{S}}(\omega)$ (left-hand panels, solid lines). On the right panel, we show $\chi_{\mathrm{S}}^{-1}$ (solid) and $\left(\chi^{A}\right)^{-1}$ (dashed) that arises from application of an adiabatic kernel $f_{\text {HXC }}^{A}$ as indicated. The dotted lines show the true inverse kernel $\chi^{-1}$ (from Eq. 19 with $q=1$, see text)

\section{DRESSED TDDFT FOR RESONANCES ARISING FROM DOUBLE EXCITATIONS}

We are interested in the frequency-range near a resonance that arises when the energy of a bound doubleexcitation lies above the single-ionization threshold. As argued in the previous section, the KS response function displays no resonance, and an adiabatic kernel cannot generate one. In this section we derive the form of the kernel that is required in order to capture this kind of res- onance, based on Fano's approach. We again stay within the assumption of weak interaction, where the dominant coupling between the KS states near the frequencies of interest, is the resonant coupling of the bound doubleexcitation with the continuum states. We again assume an isolated resonance: one discrete state coupling to one continuum.

Eq. 19 relates the imaginary part of the true response function to that of the KS response function via the Fano lineshape. Our job is now to use Eq. 13 to find the implied structure of the xc kernel.

A first simplification is that $q(\mathbf{r})^{2}=1$. This follows from the sum-rule Eq. 5 . Within the assumption that $|i\rangle$ can be approximated by the KS ground-state, then the RHS must be zero, since $\hat{T}$ is a one-body operator and $\Phi_{\mathrm{b}}$ differs from the KS ground-state by two orbitals. That $q^{2}=1$ is consistent with the oscillator strength sum-rule (eg. Ref. 34]): both the KS system and the interacting system satisfy the oscillator strength sum-rule. As the KS spectrum does not contain the resonance arising from a doubly-excited state, this suggests that the integrated area under the Fano lineshape factor should be zero. That is, the line-shape should be antisymmetric, so $q(\mathbf{r})$ should be \pm 1 .

Examination of Eq 4 reveals that $q$ must be positive for our case: the first term in the numerator on the right is zero by the above argument, while the second term divided by the denominator is positive [30], because $\left\langle\Phi_{E^{\prime}}|T| i\right\rangle$ grows larger than $\left\langle\Phi_{E}|T| i\right\rangle$ where $E-E^{\prime}>0$.

So we may conclude for the case of a double-excitation resonance, in the weak-interaction limit, that the imaginary part of the response function is given by:

$$
\Im \chi(\omega)=\frac{\left(\omega-\omega_{r}+\Gamma / 2\right)^{2}}{\left(\omega-\omega_{r}\right)^{2}+(\Gamma / 2)^{2}} \Im \chi_{\mathrm{s}}(\omega)
$$

where $\Gamma$ and $\omega_{r}$ are given by Eqs. (3) and (2), with $V_{\mathrm{cpl}}$ given by Eq. (18). An immediate implication is that the spatial-dependence is unchanged: this is true only within the approximations stated above.

Returning to our search for $f_{\mathrm{HXC}}$, Eq. 13 requires that we invert the response function. For this, we need to first calculate its real part, which we may obtain from the principle-value integral, Eq. (20). Subtracting out $\Im \chi_{\mathrm{S}}$ from $\Im \chi$, we write

$\Re \chi(\omega)=\Re \chi_{\mathrm{S}}(\omega)+\frac{2 \Gamma}{\pi} P \int_{0}^{\infty} \frac{\omega^{\prime}\left(\omega^{\prime}-\omega_{r}\right) \Im \chi_{\mathrm{S}}\left(\omega^{\prime}\right)}{\left(\omega^{\prime 2}-\omega^{2}\right)\left(\left(\omega-\omega_{r}\right)^{2}+(\Gamma / 2)^{2}\right)} d \omega^{\prime}$

In the spirit of the Fano analysis, we assume that the KS orbitals are slowly-enough varying in frequency, that $\Im \chi_{\mathrm{S}}$ can be pulled out of the principle-value integral. That is, that $\Im \chi_{\mathrm{S}}$ is relatively flat in the region of the resonance; further away from the resonance the integral vanishes as the lineshape factor decays rapidly. We obtain 
$\Re \chi(\omega)=\Re \chi_{\mathrm{S}}(\omega)+\left(\frac{\left(\frac{\Gamma^{2}}{2}(\Gamma / 2)^{2}+\left(\omega^{2}+\omega_{r}^{2}\right)\right)\left(1+\frac{2}{\pi} \tan ^{-1}\left(2 \omega_{r} / \Gamma\right)\right)-\frac{\Gamma}{2 \pi} \omega_{r}\left(\omega^{2}-\omega_{r}^{2}-(\Gamma / 2)^{2}\right) \ln \left(\frac{\omega^{2} / 4}{\omega_{r}^{2}+(\Gamma / 2)^{2}}\right)}{\left(\left(\omega-\omega_{r}\right)^{2}+\left(\frac{\Gamma}{2}\right)^{2}\right)\left(\left(\omega+\omega_{r}\right)^{2}+\left(\frac{\Gamma}{2}\right)^{2}\right)}\right) \Im \chi_{\mathrm{S}}(\omega)$

Consistent with our assumptions, we take $\Gamma<<\left(\omega_{r}-I\right)$, and considering frequencies $\omega$ near $\omega_{r}$, we obtain, after some algebra,

$$
\Re \chi(\omega)=\Re \chi_{\mathrm{S}}(\omega)+\frac{\Gamma^{2} / 2}{\left(\omega-\omega_{r}\right)^{2}+\left(\frac{\Gamma}{2}\right)^{2}} \Im \chi_{\mathrm{S}}(\omega)
$$

Putting Eqs. 25 and 22 together, we have

$$
\chi=\chi_{\mathrm{S}}+\frac{\Gamma\left(\Gamma / 2+i\left(\omega-\omega_{r}\right)\right)}{\left(\omega-\omega_{r}\right)^{2}+\left(\frac{\Gamma}{2}\right)^{2}} \Im \chi_{\mathrm{S}}
$$

The complex lineshape on the right relates the interacting response function to the non-interacting one with a dramatic resonance structure. The cross-section obtained from the imaginary part, reproduces the Fano formula.

One should not be alarmed by the poles in our approximate $\chi$ in the upper half plane, given that the exact $\chi$ should be analytic in the upper half plane, and its inverse $\chi^{-1}$ analytic for $\Im(\omega)>0$. But our approximate kernel holds only for real frequencies, moreover, for frequencies in the restricted range near the resonance.

We now use Eq. (13) to extract the frequencydependent kernel. Subtracting the inverse of Eq. 26 from that of $\chi_{\mathrm{s}}^{-1}$ (Eq. 13) we find the Hartree-exchangecorrelation kernel to be

$f_{\mathrm{HXC}}(\omega)=\chi_{\mathrm{S}}^{-1}(\omega)-\left(\chi_{\mathrm{S}}(\omega)+\frac{\Gamma\left(\Gamma / 2+i\left(\omega-\omega_{r}\right)\right)}{\left(\omega-\omega_{r}\right)^{2}+\left(\frac{\Gamma}{2}\right)^{2}} \Im \chi_{\mathrm{S}}(\omega)\right)$

In Eq. 27. the spatial-dependence has been omitted for clarity, i.e. all arguments of $f_{\mathrm{HXC}}$ and $\chi_{\mathrm{S}}$ are understood to be $\left(\mathbf{r}, \mathbf{r}^{\prime}, \omega\right)$. We call this the Fano TDDFT kernel, in that it reproduces exactly the Fano lineshape relative to the KS spectrum, when a bound double-excitation lies in the continuum. Its frequency-dependence is essential, as demonstrated by the sketches of the previous section. For illustration, we plot the real and imaginary parts of this in Figure 3, where we take $\chi_{\mathrm{S}}(\omega) \approx \frac{1}{\sqrt{\omega+1}}+\frac{i}{\sqrt{\omega-1}}$ as in the earlier plots, $\Gamma=0.1$ and $\omega_{r}=3$.

This kernel accounts only for the coupling of the KS bound state with the continuum, which is the dominant effect near the resonance. It becomes exact in the limit of weak interaction, for an isolated and narrow resonance, where $\Gamma$ is the smallest energy scale of the system.

We may also include this kernel on top of an adiabatic kernel, in order to account also for mixing of nonresonant single excitations that adiabatic TDDFT may capture well. For the case that the resonance arising from the double-excitation is well-isolated from any other resonances in the system, modifying Eq. 26, we assert the

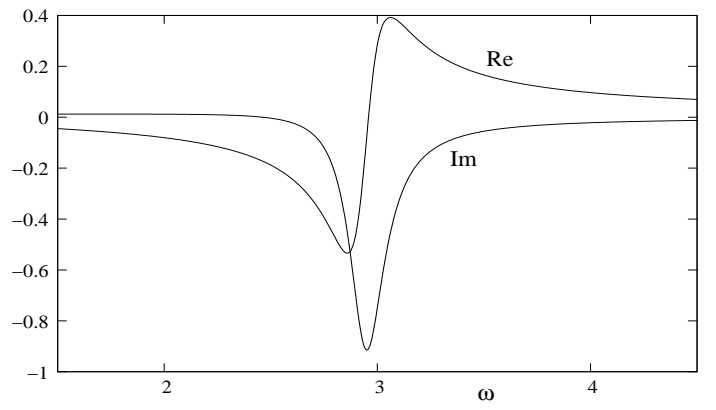

FIG. 3: Sketch of the Fano TDDFT kernel, Eq.27, that reproduces Fano's lineshape for the autoionizing resonance arising from a double-excitation.

dressed response-function approximation:

$$
\chi=\chi^{A}+\frac{\Gamma\left(\Gamma / 2+i\left(\omega-\omega_{r}\right)\right)}{\left(\omega-\omega_{r}\right)^{2}+\left(\frac{\Gamma}{2}\right)^{2}} \Im \chi^{A}
$$

where $\chi^{A}(\omega)$ is the interacting response function computed using an adiabatic xc kernel. At frequencies moving away from the resonance arising from the doubleexcitation, our dressed response function reduces to the adiabatic response function, $\chi^{A}(\omega)$; thus our dressed response function leaves untampered the usual reasonably accurate response that adiabatic TDDFT gives for single-excitation states. The corresponding dressed cross-section, obtained from its imaginary part, yields

$$
\sigma(\omega)=\frac{\left(\omega-\omega_{r}+\Gamma / 2\right)^{2}}{\left(\omega-\omega_{r}\right)^{2}+(\Gamma / 2)^{2}} \sigma^{A}(\omega)
$$

From Eq. 28 we derive the dressed kernel:

$$
\begin{gathered}
f_{\mathrm{HXC}}(\omega)=f_{\mathrm{HXC}}^{A}- \\
\left.\left(\chi^{A}\right)^{-1}\left(\left(1+\left(\chi^{A}\right)^{-1} \Im \chi^{A} \frac{\Gamma\left(\Gamma / 2+i\left(\omega-\omega_{r}\right)\right)}{\left(\omega-\omega_{r}\right)^{2}+\left(\frac{\Gamma}{2}\right)^{2}}\right)^{-1}-1\right) 30\right)
\end{gathered}
$$

In practise, computing cross-sections using our dressed TDDFT would proceed very simply: First an adiabatic calculation would be run, as in Refs. 12, 13, 14, 15, 16, 17]. Then where a bound-double excitation is known to lie (for example, by summing KS orbital frequencies), utilize the relevant KS orbitals in Eqs. (3) and (2), to find the width $\Gamma$ and shift of the resonance position $F(E)$ that appears in $\omega_{r}$. Then modify the cross-section computed using an adiabatic approximation by the lineshape (Eq. 29). That is, in practise, if interested in computing the cross-section, we would not need to utilize Eq. 30 
directly, instead we would use Eq. 29 Instead, Eq. 30 and Eq. 27 are of fundamental interest here: it is the xc kernel for which approximations are needed in TDDFT, and these equations reveal the form it requires, in order to reproduce the Fano resonance arising from a doubleexcitation.

\section{A. Model example}

We illustrate our results on a simple model involving two electrons in one-dimension living in an external potential of the form:

$$
v_{\text {ext }}(x)=-\frac{U_{0}}{\cosh ^{2}(\alpha x)}-\beta \sqrt{\left(1-\tanh ^{2}(x)\right)^{3}}
$$

We choose values of parameters $U_{o}, \alpha, \beta$ such that there are (at least) two bound single-particle states in the noninteracting problem. A sketch is shown in Figure 4 A double-excitation to the first excited orbital is shown on the left; this has energy $2 \epsilon$ where $\epsilon$ is the energy difference between the single-particle orbitals. This energy $2 \epsilon$ exceeds the single-ionization threshold for this system, and lies in the continuum; therefore the state on the left is degenerate with a single-excitation to the continuum, indicated on the right. When electron-interaction is turned on, an isolated resonance is created, of the type to which our formula and analysis applies.
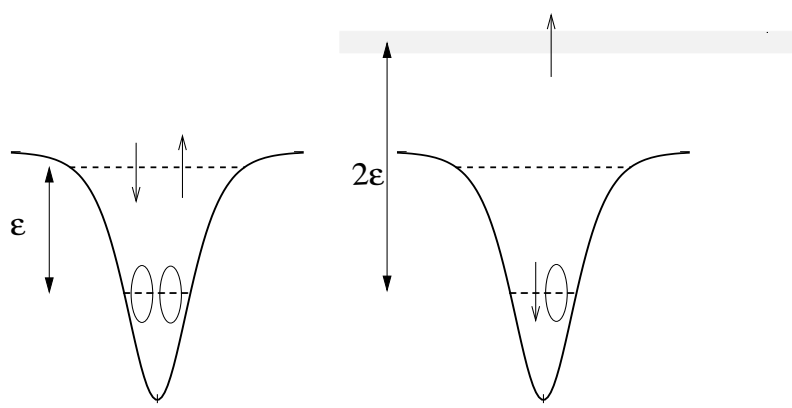

FIG. 4: Sketch of model potential, indicating the two excited non-interacting states, one a double-excitation that is bound (on the left) and the other a single-excitation that is unbound (on the right). Allowing the electrons to interact couples these degenerate states, creating a resonance.

We first find the KS potential and spectrum. We choose a weak delta-function interaction:

$$
V_{\mathrm{ee}}=\lambda \delta\left(x-x^{\prime}\right), \quad \lambda<1 .
$$

For small enough $\lambda$, exchange dominates over correlation. We use the exact-exchange approximation for two electrons:

$$
v_{\mathrm{XC}}=-v_{\mathrm{H}} / 2=-\lambda n(x) / 2
$$

The KS potential follows as:

$$
v_{\mathrm{S}}(x)=v_{\mathrm{ext}}+v_{\mathrm{H}}+v_{\mathrm{XC}}=-\frac{U_{0}}{\cosh ^{2}(\alpha x)}
$$

if we take $\beta=\lambda$ in Eq. 31. The exact one-electron eigenstates and energies of $v_{\mathrm{S}}$ (an "Eckart well") can be found in many quantum mechanics textbooks. The parameters $U_{0}, \alpha$ are chosen such that there are at least two bound one-particle states: we chose $U_{0}=1.875$, and $\alpha=1$, which places the non-interacting bound orbital energies at $\epsilon_{0}=-1.125$ and $\epsilon_{1}=-0.125$. From the one-electron orbitals we calculate the KS response function $\chi_{\mathrm{s}}$ and dipole moment, shown as the dashed line in Figure 5 , after placing two electrons in the lowest orbital. As expected, the dipole moment to the continuum states is smooth and gently decaying.

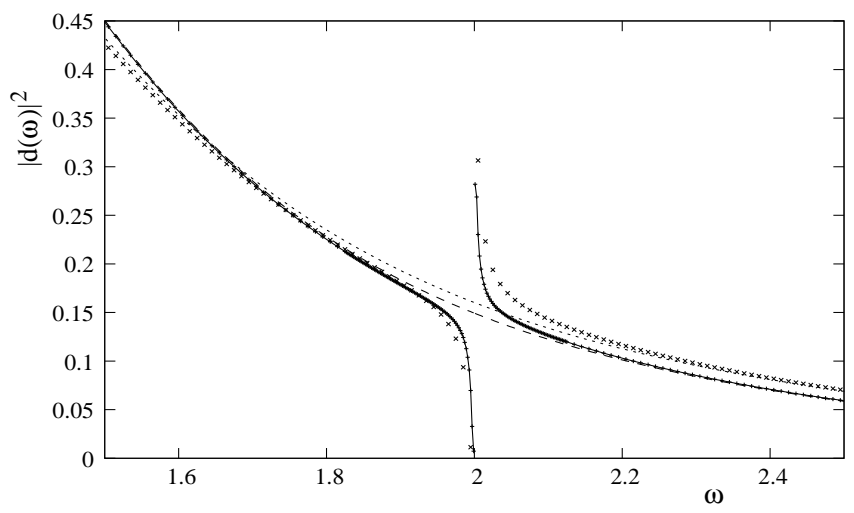

FIG. 5: The square of the dipole moment: Dashed line is KS, dotted line is the adiabatic TDDFT (exact-exchange), solid line is the resonance with the Fano lineshape as would be obtained with the Fano TDDFT kernel Eq. 27 and the (x) are our dressed kernel used in conjunction with the adiabatic approximation, Eq. 30

We now consider the TDDFT spectra, and will compute everything only to first-order in the interaction strength, $\lambda$, whose numerical value we take as 0.2 in the calculations. We first apply an adiabatic kernel, and again choose exact-exchange for this:

$$
f_{\mathrm{XC}}^{A}\left(x, x^{\prime}\right)=f_{\mathrm{X}}^{A}\left(x, x^{\prime}\right)=-\lambda \delta\left(x-x^{\prime}\right) / 2
$$

For the cases where the states are mixtures of single excitations, exchange effects dominate in the weakinteraction limit, being of $O(\lambda)$ while correlation is $O\left(\lambda^{2}\right)$; so for small values of $\lambda, f_{\mathrm{x}}^{A}$ is expected to be quite accurate for energies and oscillator strengths of states of single-excitation character. Then

$$
\chi^{A}\left(\mathbf{r}, \mathbf{r}^{\prime}, \omega\right)=\chi_{\mathrm{s}}\left(\mathbf{r}, \mathbf{r}^{\prime}, \omega\right)+\frac{\lambda}{2} \int \chi_{\mathrm{s}}\left(\mathbf{r}, \mathbf{r}_{1}, \omega\right) \chi\left(\mathbf{r}_{1}, \mathbf{r}^{\prime}, \omega\right) d^{3} r_{1}
$$

where, on the right, we may replace $\chi$ with $\chi_{\mathrm{s}}$ to get the $O(\lambda)$ adiabatic TDDFT spectrum. In one-dimension, from Eqs. 7 and 10 ,

$$
|d(\omega)|^{2}=-\frac{1}{\pi} \int x \Im \chi\left(x, x^{\prime}, \omega\right) x^{\prime} d x d x^{\prime}
$$


The KS version of Eq. 15 simplifies to

$$
\Im \chi_{\mathrm{S}}\left(x, x^{\prime}, \omega\right)=-\pi \phi_{0}(x) \phi_{0}\left(x^{\prime}\right) \phi_{\epsilon=\omega+\epsilon_{0}}(x) \phi_{\epsilon=\omega+\epsilon_{0}}\left(x^{\prime}\right)
$$

Using Eq. 38 and its principal-value integral to get the real part, Eq. 37 finally gives

$$
\begin{gathered}
\left|d^{A}(\omega)\right|^{2}=\left|d_{\mathrm{S}}(\omega)\right|^{2}+2 \lambda d_{\mathrm{S}}(\omega) \times \\
\left.\int d x_{1} \phi_{0}^{2}\left(x_{1}\right) \phi_{\epsilon}\left(x_{1}\right) P \int d_{\mathrm{S}}\left(\epsilon^{\prime}\right) \phi_{\epsilon^{\prime}}\left(x_{1}\right) \frac{\epsilon^{\prime}-\epsilon_{0}}{\omega^{2}-\left(\epsilon^{\prime}-\epsilon_{0}\right)^{2}} d \epsilon^{\prime} 39\right)
\end{gathered}
$$

This adiabatic dipole moment is the dotted line in Fig 5 . it smoothly shifts the KS spectrum, redistributing oscillator strength from the lower to higher frequencies of the KS spectrum, but is a small and smooth correction.

Applying now the frequency-dependent kernel Eq. 27 reveals the resonance (solid line), while the dressed kernel Eq. 30 (x points) incorporates corrections from the adiabatic approximation as well as capturing the resonance. As mentioned in the previous section, in practise, we utilize Eq. 29, evaluating $\Gamma$ and $\omega_{r}$ using the KS orbitals.

\section{CONCLUSIONS AND OUTLOOK}

We have discussed the implications of Fano's resonance formula for the xc kernel of TDDFT. In the case of a narrow isolated resonance involving one discrete state and one continuum, and weak interaction, we derived the Fano-equivalent formula for the imaginary part of the TDDFT response function (Eq. 19). We illustrated how a frequency-independent kernel applied to a largely frequency-independent KS response function, actually yields dramatic frequency-dependent resonant structures for the case of a bound single-excitation, but yields no structure in the case of a bound double-excitation.

Within our assumptions, we derived the exact form of the frequency-dependent kernel that is needed for the latter case: we call this the Fano TDDFT kernel (Eq. 27), in the sense that it exactly reproduces Fano's lineshapes, when applied to the KS density-response function. We then asserted a dressed frequency-dependent kernel (Eq. 30), that accounts for the Fano effect on top of an adiabatic approximation. The form of these kernels is of fundamental interest for TDDFT. In practise, we propose one computes the resonance width $\Gamma$ and position $\omega_{r}$ using the appropriate KS orbitals; compose from them the Fano lineshape (Eq. 29) and thereby modify the adiabatic spectrum. How this will work in practise for molecules of interest, remains to be tested.

In this paper we considered the simplest case of a resonance arising from a bound double excitation: that is, when the resonance is isolated from all others and involves only one continuum and one discrete state. We have not considered the interaction of resonances 39] that arises in most systems, nor the computation of individual branching ratios when several channels are involved [33]. The present work is only a first step in uncovering how the exact xc kernel captures resonances in the general case, and how to approximate it in practise.

We gratefully acknowledge financial support from the National Science Foundation NSF CHE-0547913, and a Research Corporation Cottrell Scholar Award.
[†] Present Address: Department of Physics and Astronomy, Rutgers, The State University of New Jersey, 136 Frelinghuysen Road, Piscataway, NJ 08854-8019 USA

[1] M. van Faassen and K. Burke, arXiv:0901.3418v2

[2] I. Cacelli, V. Carravetta, A. Rizzo, and R. Moccia, Phys. Rep. 205, 283 (1991).

[3] C. Froese-Fischer, Comput. Phys. Commun. 14, 145 (1978).

[4] H. P. Saha, J. Phys. B 39, 1209 (2006).

[5] J. A. Sheehy, T.J. Gill, G.L. Winstead, R.E. Farren, and P.W. Langhoff, J. Chem. Phys. 91, 1796 (1989).

[6] L. Kim and C. Greene, Phys. Rev. A.38, 2361 (1988).

[7] Sajeev and N. Moiseyev, Phys. Rev. B. 78, 075316 (2008).

[8] R. Santra and L. S. Cederbaum, J. Chem. Phys. 115, 6853 (2001).

[9] Y. Sajeev, R. Santra and S. Pal, J. Chem. Phys. 123, 204110 (2005).

[10] P. Lin and R. R. Lucchese, J. Chem. Phys. 113, 1843 (2000).

[11] I. Cacelli, R. Moccia, and A. Rizzo, Chem. Phys. 252, 67 (2000).

[12] M. Stener, P. Decleva, and A. Lisini, J. Phys. B. 28, 4973 (1995).
[13] G. Fronzoni, M. Stener, and P. Decleva, J. Chem. Phys. 118, 10051 (2003).

[14] G. Fronzoni, M. Stener, and P. Decleva, Chemical Physics 298, 141 (2004).

[15] M. Stener, G. Fronzoni, P. Decleva, J. Chem. Phys. 122, 234301 (2005).

[16] M. Stener, D. Toffoli, G. Fronzoni, and P. Decleva, J. Chem. Phys. 124, 114306 (2006).

[17] M. Stener, D. Toffoli, G. Fronzoni, and P. Decleva, Theor. Chem. Acc. 117, 943 (2007).

[18] E. Runge and E. K. U. Gross, Phys. Rev. Lett. 52, 997 (1984).

[19] E.K.U. Gross, J.F. Dobson, and M. Petersilka, Topics in Current Chemistry, 181, 81 (1996).

[20] Time-Dependent Density Functional Theory eds. M.A.L. Marques, F. Nogueira, A.Rubio, K.Burke, C.A. Ullrich, and E.K.U. Gross (Springer, Berlin, 2006)

[21] M. Petersilka, U.J. Gossmann, and E.K.U. Gross, Phys. Rev. Lett. 76, 1212 (1996).

[22] M.E. Casida, in Recent developments and applications in density functional theory, ed. J.M. Seminario (Elsevier, Amsterdam, 1996).

[23] F. Furche and R. Ahlrichs, J. Chem. Phys. 117 (2002), 7433; J. Chem. Phys. 121 (2004), 12772 (E). 
[24] N. Spallanzani, C. A. Rozzi, D. Varsano, T. Baruah, M. Pederson, F. Manghi A. Rubio, arXiv:0901.3052 1 [condmat.mtrl-si]

[25] M. A. L. Marques, X. Lopez, D. Varsano, A. Castro, and A. Rubio, Phys. Rev. Lett. 90, 258101 (2003).

[26] E. Tapavicza, I. Tavernelli, U. Roethlisberger, C. Filippi, M. E. Casida, J. Chem. Phys., J. Chem. Phys. 129, 124108 (2008)

[27] N.T. Maitra, F. Zhang, R.J. Cave and K. Burke, J. Chem. Phys. 120, 5932 (2004).

[28] G. Mazur and R. Wlodarczyk, J. Comp. Chem. (2008).

[29] R.J. Cave, F. Zhang, N.T. Maitra, and K. Burke, Chem. Phys. Lett. 389, 39 (2004).

[30] U. Fano, Phys. Rev. 124, 1866 (1961).

[31] U. Fano, Nuovo Cimento 12, 156 (1935).

[32] U. Fano and J. W. Cooper, Phys. Rev. 137, 1364 (1965).
[33] A. Starace, Phys Rev. A 16, 231 (1977).

[34] H. Friedrich, Theoretical Atomic Physics, (SpringerVerlag, Berlin (1991))

[35] G. F. Gabriele and G. Vignale, Quantum Theory of the Electron Liquid, (Cambridge University Press, United Kingdom, 2005).

[36] N.T. Maitra, K. Burke, and C. Woodward, Phys. Rev. Lett. 89, 023002 (2002) 4).

[37] D.J. Tozer and N.C. Handy, Phys. Chem. Chem. Phys. 2, 2117 (2000).

[38] M. E. Casida, J. Chem. Phys. 122, 054111 (2005).

[39] J. P. Connerade and A.M. Lane, Rep. Prog. Phys. 51 1439 (1988). 